\title{
A Randomized Controlled Clinical Trial on Therapeutic Effects of Teicoplanin and Vancomycin after Cardiac Surgery due to MRSA infective endocarditis in ICU Patients
}

\author{
Batoul Khoundabi ${ }^{1,2}$, Neda Behzadnia ${ }^{3,}$, Zargham Hossein Ahmadi ${ }^{3}$, Mandana Chitsazan ${ }^{4}$, Payam Tabarsi ${ }^{5}$, \\ Hamidreza Jamaati ${ }^{4}$, Alireza Bahadorbeigi ${ }^{4}$ and Seyed MohammadReza Hashemian ${ }^{4}$ \\ ${ }^{1}$ Iran Helal Institute of Applied Science and Technology, Tehran, Iran \\ 2 Research Center for Health Management in Mass Gathering, Red Crescent Society of the Islamic Republic of Iran, Tehran, Iran \\ ${ }^{3}$ Lung Transplant Research Center, National Research Institute of Tuberculosis and Lung Diseases, Shahid Beheshti University of Medical Sciences, Tehran, Iran \\ ${ }^{4}$ Chronic Respiratory Diseases Research Center, National Research Institute of Tuberculosis and Lung Diseases, Shahid Beheshti University of Medical Sciences, \\ Tehran, Iran \\ ${ }^{5}$ Clinical Tuberculosis and Epidemiology Research Center, National Research Institute of Tuberculosis and Lung Diseases, Shahid Beheshti University of Medical \\ Sciences, Tehran, Iran
}

* Corresponding author: Neda Behzadnia, Lung Transplant Research Center, National Research Institute of Tuberculosis and Lung Diseases, Shahid Beheshti University of Medical Sciences, Tehran, Iran. Email: nedabehzadnia@yahoo.com

Received 2020 October 27; Revised 2020 November 22; Accepted 2020 December 12.

\begin{abstract}
Background: Infective endocarditis (IE) is a relatively rare disease but with significant rates of morbidity and mortality. Vancomycin and teicoplanin are bacteriostatic glycopeptide antibiotics used for the treatment of methicillin-resistant Staphylococcus aureus (MRSA) infections.

Objectives: The current study aimed to compare the efficacy and adverse effects of vancomycin and teicoplanin in the treatment of patients with MRSA-IE.

Methods: This parallel, randomized, and controlled trial study was carried out on the efficacy and safety of teicoplanin versus vancomycin in the treatment of MRSA endocarditis within August 2012 and April 2017. The present study recruited adult patients with a definite or possible diagnosis of IE based on the Modified Duke Criteria. Vancomycin was intravenously administered at a dose of $30 \mathrm{mg} / \mathrm{kg} / \mathrm{day}$ in two doses. Teicoplanin was administered at a loading dose of $6-12 \mathrm{mg} / \mathrm{kg}$ every $12 \mathrm{~h}$ in four doses and then continued once a day.

Results: Out of 86 patients with suspected IE, 66 patients were randomly assigned to the vancomycin $(n=33)$ and teicoplanin $(n=33)$ groups. The mean age values of the study subjects were $41 \pm 11.8$ and $39 \pm 13.1$ years in the vancomycin and teicoplanin groups, respectively. In addition, 27 patients (81.8\%) in the vancomycin group met the criteria for microbiological cure, compared to 25 subjects $(89.3 \%)$ in the teicoplanin group. In this regard, the observed difference was not statistically significant $(\mathrm{P}=0.41)$. Overall, the patients in the vancomycin group experienced more adverse events in comparison to those of the teicoplanin group $(\mathrm{P}=0.04)$. The rate of acute kidney injury over time, especially in the first week of therapy, was higher in the vancomycin group than that reported for the teicoplanin group $(\mathrm{P}=0.05)$.

Conclusion: It was concluded that the administration of vancomycin or teicoplanin does not significantly change the outcome of patients undergoing empirical treatment for MRSA-IE.

Keywords: Infective endocarditis, Vancomycin, Teicoplanin
\end{abstract}

\section{Background}

Infective endocarditis (IE) is a relatively rare disease but with significant rates of morbidity and mortality. In 2010, IE caused more than 1.5 million disability-adjusted life years globally (1). The epidemiology of IE is variable both in terms of incidence and causative agents. A 2013 systematic review of studies mainly on industrialized countries demonstrated a trend toward staphylococci (mainly Staphylococcus aureus) in comparison to streptococci (mainly Streptococcus viridans) as the predominant causative agent of IE in recent decades (2). A similar trend has been observed in Iran, which might be attributed to an increase in intravenous drug abusers $(3,4)$.

Although the data regarding the prevalence of identified organisms involved in IE differ, S. aureus is considered a major agent and in many studies recognized to be the most prevalent $(2,4-7)$. Inhospital mortality is reported within the range of 14$24 \%(6-11)$, and 1-year mortality is as high as $50 \%$ (12). Furthermore, IE due to $S$. aureus infection is associated with an increased rate of mortality $(7,13)$. Methicillin-resistant Staphylococcus aureus (MRSA) is becoming a more frequent subgroup of $S$. aureus endocarditis worldwide and is associated with persistent bacteremia (14).

The goal of IE treatment is to eradicate the foci of infection, including antimicrobial therapy with or without surgery. The treatment is usually initiated empirically immediately after obtaining blood samples and tailored to the identified organism after receiving culture and antimicrobial susceptibility results (15). The decision on the selection of an empirical therapy regimen is diverse based on the

Copyright (C) 2021, Author(s). This is an open-access article distributed under the terms of the Creative Commons Attribution-NonCommercial 4.0 International License (http://creativecommons.org/licenses/by-nc/4.0/) which permits copy and redistribute the material just in noncommercial usages, provided the original work is properly cited 
patient characteristics, such as age, intravenous drug abuse, presence of prosthetic heart valve, prior antimicrobial therapy, and comorbidities (15).

\section{Objectives}

Vancomycin and teicoplanin are bacteriostatic glycopeptide antibiotics used for the treatment of MRSA infections, including MRSA-IE, and patients allergic to penicillin who are infected with methicillin-sensitive $S$. aureus. Teicoplanin, which is not available in the United States, has been shown to have similar efficacy in comparison to vancomycin in the treatment of MRSA infections, but with fewer side effects, including nephrotoxicity, skin rash, and red man syndrome $(16,17)$. However, to the best of our knowledge, a limited number of studies were carried out on the comparison between these two drugs in MRSA-IE patients (18). Therefore, the current study aimed to compare the efficacy and adverse effects of vancomycin and teicoplanin in the treatment of patients with MRSA-IE.

\section{Methods}

\subsection{Study design}

This parallel, randomized, controlled trial study was carried out on the efficacy and safety of teicoplanin versus vancomycin in the treatment of MRSA endocarditis within August 2012 to April 2017. The current study was conducted by a multidisciplinary team consisting of intensivists, cardiologists, internists, and infectious disease specialists of Masih Daneshvari Hospital in Tehran, Iran. The study protocol was approved by the Research Ethics Committee of Shahid Beheshti University of Medical Sciences, Tehran, Iran. The trial was registered at the Iranian Registry of Clinical Trials (IRCT2016022220592N4).

\subsection{Study population}

The present study recruited adult patients with a definite or possible diagnosis of IE based on the Modified Duke Criteria (19). The participants were patients referred from other hospitals, admitted to the general and surgical wards or intensive care unit of the hospital for other reasons, and admitted to the emergency department of the hospital. In addition, patients in whom further workup ruled out the diagnosis of IE, whose blood culture revealed organisms other than MRSA, and with known allergy to either of the study drugs were excluded from the study. Patients with confirmed endocarditis, over the age of 65 years, and acute kidney injury (AKI) at presentation were also excluded and received linezolid as the main antimicrobial regimen.

The study subjects were provided with accurate information about the study protocol. Then, informed written consent was obtained from the patients. After a complete physical examination, comprehensive laboratory tests were performed, and blood cultures were drawn. Antibiotic susceptibility testing was performed for all the cultures. All the patients underwent transthoracic echocardiography, carried out by experienced cardiologists. Transesophageal echocardiography was performed when indicated.

\subsection{Study interventions}

The eligible patients were randomly assigned to receive either teicoplanin or vancomycin.

\subsection{Randomization and masking}

A central computer-generated random number list was used to allocate the patients $(1: 1)$ to the treatment with teicoplanin or vancomycin. The allocation was concealed in sealed numbered envelopes that were opened by a nurse before the initiation of therapy. The participants, study investigators, research coordinators, outcome assessors, and data analysts were blinded to the treatment allocation. The nurses and attending physicians providing care to the patients who were not blinded; however, they had no access to study data and results.

Vancomycin was intravenously administered at a dose of $30 \mathrm{mg} / \mathrm{kg} /$ day in two doses. Teicoplanin was administered at a loading dose of $6-12 \mathrm{mg} / \mathrm{kg}$ every $12 \mathrm{~h}$ in four doses and then continued once a day. For the patients with prosthetic valves, combined antibiotic treatment with intravenous rifampin $(1200$ $\mathrm{mg} /$ day in two doses for at least 6 weeks) and intravenous gentamicin $(3 \mathrm{mg} / \mathrm{kg} /$ day in two doses for 2 weeks) was administered. The treatment was continued for at least 6 weeks or until the clinical and laboratory parameters of infection returned to normal and there was no sign of vegetation on followup echocardiography.

Antibiotics were intravenously administered in the hospital. Monitoring of the serum levels of vancomycin was not possible as a result of international sanctions against Iran, including no access to laboratory kits. According to the guidelines of the American Heart Association and European Society of Cardiology, the patients with uncontrolled infection, heart failure, and susceptibility to embolic events underwent surgical intervention $(15,20)$.

\subsection{Outcomes}

The primary outcomes were microbiological cure defined as a negative blood culture which was previously positive and clinical cure defined as a significant improvement of signs and symptoms by the end of treatment. The secondary outcomes included the modification of treatment, length of hospital stay, adverse events (including AKI, rash, and thrombocytopenia), and mortality. Renal failure is defined using the Acute Kidney Injury Network criteria (i.e., an elevation of at least $0.3 \mathrm{mg} / \mathrm{dL}$ in the 
baseline levels of creatinine, a $50 \%$ increase in two different measurements, or a urine output of lower than $0.5 \mathrm{~mL} / \mathrm{kg} / \mathrm{h}$ for over $6 \mathrm{~h}$ ) (21). Thrombocytopenia is defined as a platelet count below the lower limit of normal $(<150,000 / \mu L)$ or $25 \%$ reduction from the baseline count.

\section{Results}

Out of 86 patients screened with suspected IE, 66 subjects were randomly assigned to the vancomycin $(n=33)$ and teicoplanin $(n=33)$ groups. Five patients from the teicoplanin group were lost to follow-up since they were voluntarily transferred to other hospitals. The recruitment and study design is shown in a flowchart (Figure 1). The modification of the treatment was also carried out as vancomycin was switched to linezolid in cases of stage II and III of AKI $(n=2)$, rash $(n=5)$, and thrombocytopenia $(n=3)$. In the teicoplanin group, the dose of the antibiotic was adjusted after the occurrence of any stages of AKI (in all eight patients developing AKI). Five participants in the teicoplanin group were voluntarily opted out of the study, which was not related to the interventions.

\subsection{Baseline characteristics}

Table 1 tabulates the baseline demographics and clinical characteristics of the study subjects. In general, the baseline characteristics of the patients were compared between the two groups. Overall, the majority $(59.0 \%)$ of the patients were male in this study. The mean age values of the study participants were $41 \pm 11.8$ and $39 \pm 13.1$ years in the vancomycin and teicoplanin groups, respectively. In addition, congenital heart diseases, acquired valvular diseases, and prosthetic heart valves were observed in 8 (13.1\%), 15 (24.6\%), and 7 (11.5\%) patients, respectively. Furthermore, 15 patients (24.6\%) were intravenous drug users, and 3 patients $(4.9 \%)$ were human immunodeficiency virus (HIV) positive.

\subsection{Outcomes}

In this study, 27 patients (81.8\%) in the vancomycin group met the criteria for microbiological cure, compared to 25 subjects $(89.3 \%)$ in the teicoplanin group. In this regard, the observed difference was not statistically significant $(\mathrm{P}=0.41)$. Moreover, $26(78.8 \%)$ and $23(82.1 \%)$ patients in the vancomycin and teicoplanin groups achieved clinical cure, respectively $(\mathrm{P}=0.97)$.

\subsection{Surgery}

In the vancomycin group, 10 patients (30.3\%)

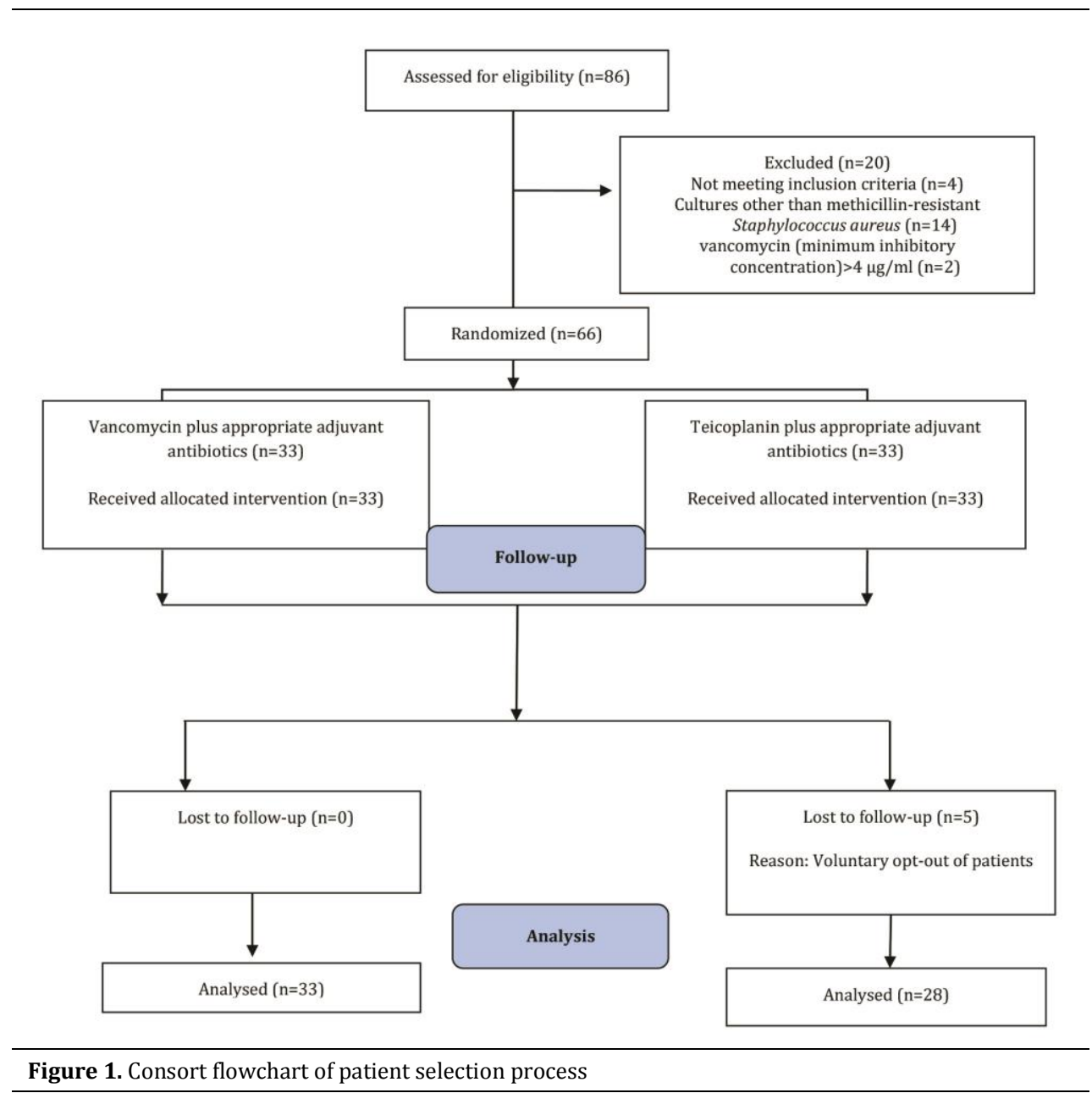




\begin{tabular}{|c|c|c|c|}
\hline Variable & Vancomycin group $(n=33)$ & $\begin{array}{l}\text { Teicoplanin group } \\
(n=28)\end{array}$ & P-value \\
\hline Age (year) & $41 \pm 11.8$ & $39 \pm 13.1$ & 0.72 \\
\hline Male & $20(60.6)$ & $16(57.1)$ & 0.45 \\
\hline Diabetes mellitus & $4(12.1)$ & $3(10.7)$ & 0.72 \\
\hline Hypertension & $6(18.2)$ & $4(14.3)$ & 0.77 \\
\hline Malignancy & $2(6.1)$ & $2(7.1)$ & 0.53 \\
\hline Coronary artery disease & $3(9.1)$ & $2(7.1)$ & 0.53 \\
\hline Congestive heart failure & $2(6.0)$ & $1(3.5)$ & 0.41 \\
\hline Congenital valvular disease & $4(12.1)$ & $4(14.3)$ & 0.59 \\
\hline Acquired valvular disease & $8(24.2)$ & $7(25.0)$ & 0.61 \\
\hline Prosthetic valve & $4(12.1)$ & $3(10.7)$ & 0.54 \\
\hline Cardiac pacemaker & $2(6.1)$ & $1(3.6)$ & 0.23 \\
\hline Short-term catheter & $3(9.1)$ & $1(3.6)$ & 0.77 \\
\hline Chronic central catheter & $1(3.0)$ & $1(3.6)$ & 0.45 \\
\hline Intravenous drug user & $8(24.2)$ & $7(25.0)$ & 0.44 \\
\hline Single valve involvement & $22(66.7)$ & $19(67.9)$ & 0.66 \\
\hline Multiple valve involvement & $11(33.3)$ & $9(32.1)$ & 0.76 \\
\hline \multicolumn{4}{|l|}{ Involved valve } \\
\hline Tricuspid valve & $19(57.6)$ & $15(53.6)$ & 0.56 \\
\hline Mitral valve & $14(42.4)$ & $12(42.9)$ & 0.63 \\
\hline Pulmonary valve & $6(18.2)$ & $4(14.3)$ & 0.73 \\
\hline APACHE II score & $9.10 \pm 3.2$ & $11.21 \pm 4.9$ & 0.11 \\
\hline Sequential organ failure assessment & $6.46 \pm 4.1$ & $6.33 \pm 3.9$ & 0.45 \\
\hline
\end{tabular}

Values shown as number (\%) unless otherwise specified

underwent surgery. Moreover, three patients (9.1\%) had a surgical valve repair or replacement as a result of uncontrolled infection, and seven subjects (20.2\%) experienced heart failure and required surgical intervention in the vancomycin group. In the teicoplanin group, eight subjects $(28.5 \%)$ underwent surgery. In addition, two patients $(6.1 \%)$ had surgical interventions due to uncontrolled infection, and six participants $(18.2 \%)$ underwent surgery due to heart failure in the teicoplanin group. The rate of surgical interventions was not statistically different between the two groups $(\mathrm{P}=0.98)$.

\subsection{Total adverse events}

Table 2 tabulates the total adverse events in this study. Overall, the patients in the vancomycin group experienced more adverse events, compared to the subjects in the teicoplanin group $(\mathrm{P}=0.04)$.

\subsection{Acute kidney injury rate and creatinine changes}

In this study, $12(36.4 \%)$ and $8(28.6 \%)$ patients in the vancomycin and teicoplanin groups developed AKI, respectively (Table 2). In this regard, the difference was not statistically significant in the baseline $(\mathrm{P}=0.51)$. However, the AKI rates over time, especially in the first and second weeks of therapy, were significantly higher in the vancomycin group than those reported for the teicoplanin group $(\mathrm{P}<0.05$; Figure 2). Figure 3 illustrates the changes in

\begin{tabular}{lccc}
\hline \multicolumn{4}{l}{ Table 2. Adverse effects in two study groups } \\
\hline & $\begin{array}{c}\text { Vancomycin } \\
\text { n (\%) }\end{array}$ & $\begin{array}{c}\text { Teicoplanin } \\
\text { n (\%) }\end{array}$ & P-value \\
\hline Acute kidney injury & $12(36.4)$ & $8(28.6)$ & 0.51 \\
Rash & $4(12.1)$ & $0(0.0)$ & 0.11 \\
Red man syndrome & $1(3.0)$ & $0(0.0)$ & 0.99 \\
Thrombocytopenia & $3(9.1)$ & $1(3.6)$ & 0.61 \\
Total adverse effects & $20(60.6)$ & $9(32.2)$ & $0.04^{*}$ \\
\hline
\end{tabular}

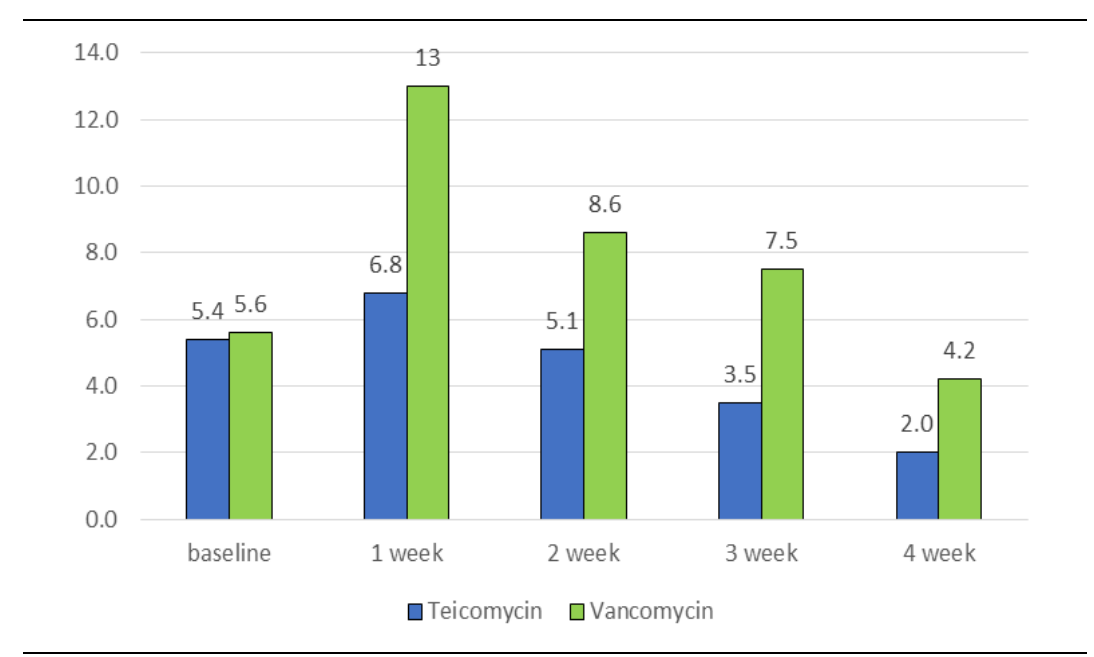

Figure 2. Changes in acute kidney injury rates during 4 weeks in two groups 


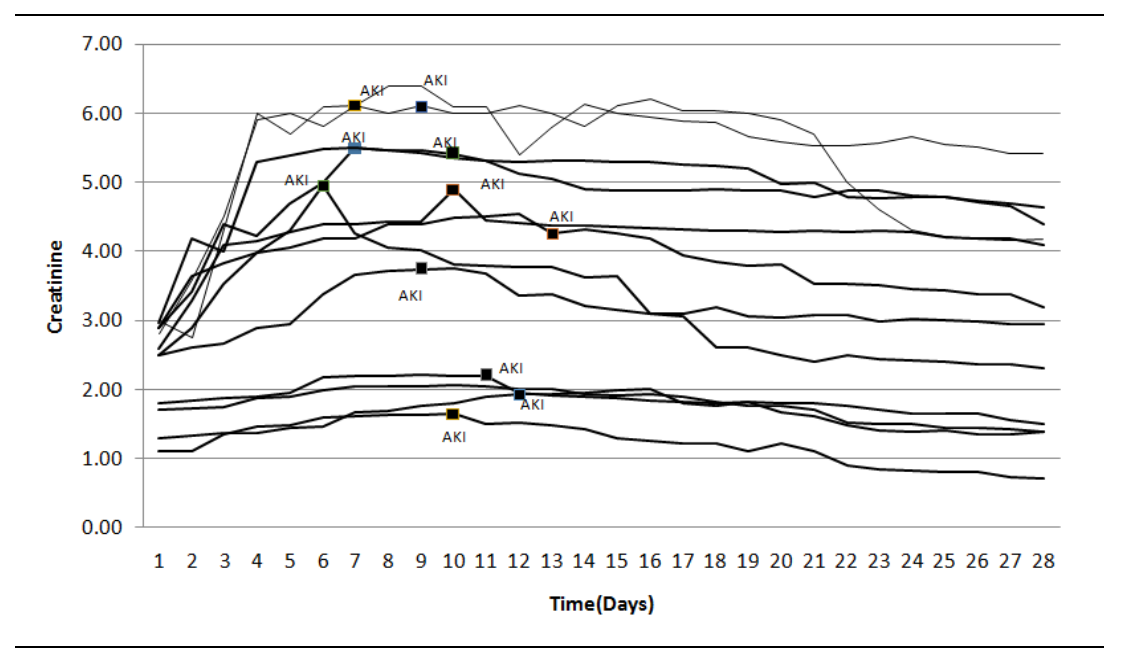

Figure 3. Changes in creatinine levels of patients with acute kidney injury

the creatinine levels of these patients. Most AKIs in both groups occurred in the first week of therapy.

Six patients in the vancomycin group required dialysis. Moreover, two and four patients underwent Continuous Renal Replacement Therapy (CRRT) and conventional hemodialysis in the vancomycin group, respectively. Four participants in the teicoplanin group required dialysis. Furthermore, one and three patients in the teicoplanin group underwent CRRT and conventional hemodialysis, respectively. The use of dialysis was not different between the two groups $(\mathrm{P}=0.88)$. In the patients with AKI in the vancomycin group, the antibiotic regimen was changed to teicoplanin (creatinine values in Figure 2 after index event are obtained during antibiotic therapy with teicoplanin).

\subsection{Rash}

In the vancomycin group, four patients developed a cutaneous rash. None of these study subjects received rifampin. One patient experienced red man syndrome in the vancomycin group. The clinicians decided to switch the antibiotic regimen in these patients to teicoplanin. No rash was observed in the teicoplanin group.

\subsection{Thrombocytopenia}

Three patients (9\%) in the vancomycin group and one subject $(3.6 \%)$ in the teicoplanin group developed thrombocytopenia. None of the thrombocytopenia cases were clinically significant, and none of them reached platelet counts below 50,000. The thrombocytopenic patients were closely monitored, and no modification was carried out in their antibiotic regimen.

\subsection{Mortality}

Seven patients $(21.3 \%)$ died in the vancomycin group. The cause of mortality in the three subjects was AKI. One participant died due to thromboembolic complications. Moreover, one patient died due to

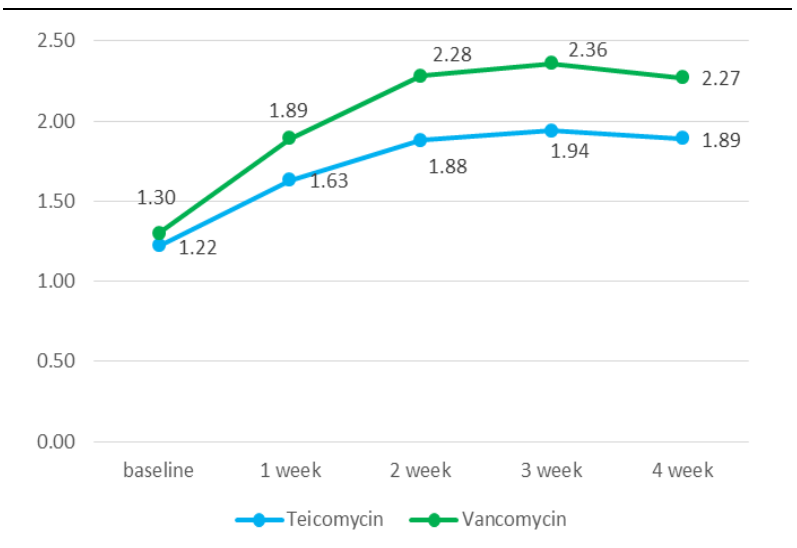

Figure 4. Sequential organ failure assessment changes during 4 weeks in two groups

disseminated intravascular coagulation (DIC), and two patients died due to refractory sepsis. In the teicoplanin group, five patients $(17.9 \%)$ died. The cause of mortality was AKI in two patients, thromboembolic complications in one participant, DIC in one case, and refractory sepsis in another subject. The overall rate of mortality was not statistically different between the two groups $(\mathrm{P}=0.74)$.

\subsection{SOFA}

There was no significant difference in the mean sequential organ failure assessment (SOFA) score of the two groups on admission (i.e., 6.33 and 6.46 in the teicoplanin and vancomycin groups, respectively $[P=0.45])$. Figure 4 depicts the SOFA trend in the subsequent weeks. At the end of the fourth week, the mean SOFA scores were 1.88 and 2.64 in the teicoplanin and vancomycin groups, respectively $(\mathrm{P}<0.05)$.

\section{Discussion}

As previously mentioned, the incidence of IE is rare; however, it is an important disease due to the 
high rates of morbidity and mortality (1). In addition to industrialized countries, Staphylococcus aureus was more frequently reported than streptococcus viridans in Iran as the predominant agent in recent decades (2). The MRSA is becoming a more frequent subgroup of $S$. aureus endocarditis worldwide (14), and empiric antibiotic therapy is based on the patient characteristics, including age, intravenous drug abuse, presence of prosthetic heart valve, prior antimicrobial therapy, and comorbidities (15). Vancomycin and teicoplanin are bacteriostatic glycopeptide antibiotics used for the treatment of MRSA-IE. The current study compared the efficacy and adverse effects of vancomycin and teicoplanin in the treatment of patients with MRSA-IE.

Lost to follow-up was reported in five cases (15\%) in the teicoplanin group due to transferring to another hospital which did not significantly affect the results. The majority (59\%) of the patients were male in this study. The mean age values of the patients were $41 \pm 11.8$ and $39 \pm 13.1$ years in the vancomycin and teicoplanin groups, respectively. Moreover, 8 patients (13.1\%) had congenital heart diseases, and 15 participants $(24.6 \%)$ had acquired valvular diseases. Furthermore, 7 subjects (11.5\%) had prosthetic heart valves, and 15 participants $(24.6 \%)$ were intravenous drug users. In addition, three patients (4.9\%) were HIV positive.

In this study, 27 (81.8\%) and 25 (89.3\%) patients in the vancomycin and teicoplanin groups met the criteria for microbiological cure, respectively. This difference was not statistically significant $(\mathrm{P}=0.41)$. This similarity in microbiological cure was in line with the findings of a study carried out by Jih-Hsin Huang and Ron-Bin Hsu (18) on the comparison of vancomycin and teicoplanin as anti-microbial agents for the treatment of MRSA-IE. In addition to this similarity, the majority of microbiological cure in both groups was significantly higher than that reported for the study by Jih-Hsin Huang and Ron-Bin Hsu. The antibiotic failure was reported in the aforementioned study with a higher rate in comparison to that of the current study which may be due to some factors, such as the severity of the disease, antibiotic resistance in each country, and antibiotic misuse in each state.

In the vancomycin group, 10 patients (30.3\%) underwent surgery, including 3 cases for a surgical valve repair or replacement as a result of uncontrolled infection and 7 subjects for surgical intervention as a treatment for IE induced heart failure. In the teicoplanin group, eight patients (28.5\%) underwent surgery, including two cases for uncontrolled infection and six subjects for heart failure. The rate of surgical interventions was not statistically different between the two groups $(\mathrm{P}=0.98)$. This result was also in line with the findings of studies carried out by Jih-Hsin Huang and Ron-Bin Hsu (18) and Martí-Carvajal AJ et al. (22).
Overall, the patients in the vancomycin group significantly experienced more adverse events, compared to the subjects of the teicoplanin group $(\mathrm{P}=0.04)$. This finding is in contrast to the results of a review study and meta-analysis conducted on vancomycin and teicoplanin by Martí-Carvajal $\mathrm{AJ}$ et al. (22). In other words, teicoplanin can be used as a reserve antibiotic for patients who have endocarditis and present with the adverse effects of vancomycin. This result is also confirmed by the findings of studies carried out by Cavalcanti AB et al. (16) and Svetitsky S (17).

In this study, 12 (36.4\%) and 8 (28.6\%) patients in the vancomycin and teicoplanin groups developed AKI, respectively; however, the difference was not statistically significant $(\mathrm{P}=0.51)$. In the patients with $\mathrm{AKI}$ in the vancomycin group, the antibiotic regimen was changed to teicoplanin which is inconsistent with the data in a study by Svetitsky S et al. (17). It may be due to the dose adjustment and infusion over an hour after the antibiotic treatment in the present study. In the vancomycin group, four patients developed a cutaneous rash, and one subject experienced red man syndrome (switched to teicoplanin). No rash was observed in the teicoplanin group. These results are approximately in line with all the findings of studies carried out on rashes, such as studies by Cavalcanti AB et al. (16) and Svetitsky S et al. (17)

Uncomplicated thrombocytopenia with platelet counts of more than 50,000 was reported in $9 \%$ and $3.6 \%$ of the subjects in the vancomycin and teicoplanin groups, respectively. This adverse effect is also confirmed by the results of a study conducted by Martí-Carvajal AJ et al. (22). In the current study, mortality was reported in seven $(21.3 \%)$ and five $(17.9 \%)$ patients in the vancomycin and teicoplanin groups, respectively. The overall rate of mortality was not statistically different between the two groups $(\mathrm{P}=0.74)$. Martí-Carvajal AJ et al. (22) confirmed that the administration of either vancomycin or teicoplanin will not affect the mortality rate similar to other factors, such as quick sequential organ failure assessment score.

\section{Conclusion}

According to the results of the current study and similar studies, it can be concluded that the administration of vancomycin or teicoplanin does not significantly change the outcome of patients undergoing empirical treatment for MRSA-IE. This finding indicates that they are similar in clinical outcomes and mortalities despite some adverse effects, such as skin rashes; therefore, teicoplanin can be considered a treatment choice in infective endocarditis. The use of teicoplanin in AKI patients receiving vancomycin made teicoplanin a drug of choice in patients with acute renal failure or 
creatinine rise. It is suggested to carry out further studies in this regard with a larger sample size.

\section{References}

1. Murray CJL, Vos T, Lozano R, Naghavi M, Flaxman AD, Michaud C, et al. Disability-adjusted life years (DALYs) for 291 diseases and injuries in 21 regions, 1990-2010: a systematic analysis for the Global Burden of Disease Study 2010. Lancet. 2012; 380(9859):2197-223. doi: 10.1016/S0140-6736(12)61689-4. [PubMed: 23245608].

2. Slipczuk L, Codolosa JN, Davila CD, Romero-Corral A, Yun J, Pressman GS, et al. Infective endocarditis epidemiology over five decades: a systematic review. PloS one. 2013; 8(12):e82665. doi: 10.1371/journal.pone.0082665. [PubMed: 24349331].

3. Alavi SM, Behdad F. Infective endocarditis among hospitalized intravenous drug user patients in the south west of Iran. PakJ Med Sci. 2010;26(3):659-62.

4. Hajihossainlou B, Heidarnia MA, Sharif Kashani B. Changing pattern of infective endocarditis in Iran: A 16 years survey. Pak J Med Sci. 2013;29(1):85-90. doi: 10.12669/pims.291.2682. [PubMed: 24353514].

5. DeSimone DC, Tleyjeh IM, Correa de Sa DD, Anavekar NS, Lahr BD, Sohail MR, et al. Temporal trends in infective endocarditis epidemiology from 2007 to 2013 in Olmsted County, MN. Am Heart J. 2015;170(4):830-6. doi: 10.1016/j.ahj.2015.07.007. [PubMed: 26386808].

6. Murdoch DR, Corey GR, Hoen B, Miro JM, Fowler Jr VG, Bayer AS, et al. Clinical presentation, etiology, and outcome of infective endocarditis in the 21st century: the international collaboration on endocarditis-prospective cohort study. Arch Intern Med. 2009;169(5):463-73. doi: 10.1001/archinternmed.2008.603. [PubMed: 19273776].

7. Selton-Suty C, Celard M, Le Moing V, Doco-Lecompte T, Chirouze C, Iung B, et al. Preeminence of staphylococcus aureus in infective endocarditis: a 1-year population-based survey. clinical infectious diseases : an official publication of the infectious diseases society of america. Clin Infect Dis 2012;54(9):1230-9. doi: 10.1093/cid/cis199. [PubMed: 22492317]

8. Hoen B, Alla F, Selton-Suty C, Beguinot I, Bouvet A, Briancon S, et al. Changing profile of infective endocarditis: results of a 1year survey in France. JAMA. 2002;288(1):75-81. doi: 10.1001/jama.288.1.75. [PubMed: 12090865].

9. Cabell CH, Jollis JG, Peterson GE, Corey GR, Anderson DJ, Sexton DJ, et al. Changing patient characteristics and the effect on mortality in endocarditis. Arch Intern Med. 2002;162(1):90-4. doi: 10.1001/archinte.162.1.90. [PubMed: 11784225].

10. Sy RW, Kritharides L. Health care exposure and age in infective endocarditis: results of a contemporary population-based profile of 1536 patients in Australia. Eur Heart J. 2010; 31(15):1890-7. doi: 10.1093/eurheartj/ehq110. [PubMed: 20453066].

11. Cresti A, Chiavarelli M, Scalese M, Nencioni C, Valentini S,
Guerrini F, et al. Epidemiological and mortality trends in infective endocarditis, a 17-year population-based prospective study. Cardiovasc Diagn Ther. 2017;7(1):27-35. doi: 10.21037/ cdt.2016.08.09. [PubMed: 28164010].

12. Habib G. Infective Endocarditis: Epidemiology, Diagnosis, Imaging, Therapy, and Prevention. 1 ed: Springer International Publishing; 2016.

13. Fedeli U, Schievano E, Buonfrate D, Pellizzer G, Spolaore P. Increasing incidence and mortality of infective endocarditis: a population-based study through a record-linkage system. $B M C$ Infect Dis. 2011;11(1):48. doi: 10.1186/1471-2334-11-48. [PubMed: 21345185].

14. Fowler VG, Miro JM, Hoen B, Cabell CH, Abrutyn E, Rubinstein E, et al. Staphylococcus aureus endocarditis: A consequence of medical progress. JAMA. 2005;293(24):3012-21. doi: 10.1001/jama.293.24.3012. [PubMed: 15972563].

15. Baddour LM, Wilson WR, Bayer AS, Fowler Jr VG, Tleyjeh IM, Rybak MJ, et al. Infective endocarditis in adults: diagnosis, antimicrobial therapy, and management of complications: a scientific statement for healthcare professionals from the american heart association. Circulation. 2015;132(15):1435-86. doi: 10.1161/CIR.0000000000000296. [PubMed: 26373316].

16. Cavalcanti AB, Goncalves AR, Almeida CS, Bugano DD, Silva E. Teicoplanin versus vancomycin for proven or suspected infection. Cochrane Database Sys Rev. 2010;(6). doi: 10.1002/14651858.CD007022.pub2. [PubMed: 20556772].

17. Svetitsky S, Leibovici L, Paul M. Comparative efficacy and safety of vancomycin versus teicoplanin: systematic review and metaanalysis. Antimicrob Agents Chemother. 2009;53(10):4069-79. doi: 10.1128/AAC.00341-09. [PubMed: 19596875].

18. Huang JH, Hsu RB. Treatment of infective endocarditis caused by methicillin-resistant Staphylococcus aureus: teicoplanin versus vancomycin in a retrospective study. Scand $j$ Infect Dis. 2008;40(6-7):462-7. doi: 10.1080/00365540701837126. [PubMed: 18584532].

19. Li JS, Sexton DJ, Mick N, Nettles R, Fowler Jr VG., Ryan T, et al. Proposed modifications to the Duke criteria for the diagnosis of infective endocarditis. Clin Infect Dis. 2000;30(4):633-8. doi: 10.1086/313753. Epub 2000 Apr 3. [PubMed: 10770721].

20. Habib G, Lancellotti P, Antunes MJ, Bongiorni MG, Casalta J-P, Del Zotti F, et al. 2015 ESC Guidelines for the management of infective endocarditisthe task force for the management of infective endocarditis of the european society of cardiology (ESC). Endorsed by: european association for cardio-thoracic surgery (EACTS), the european association of nuclear medicine (EANM). Eur Heart J. 2015;36(44):3075-128. doi: 10.1093/eurheartj/ehv319. [PubMed: 26590409].

21. Mehta RL, Kellum JA, Shah SV, Molitoris BA, Ronco C, Warnock DG, et al. Acute kidney injury network: report of an initiative to improve outcomes in acute kidney injury. Crit care. 2007;11(2):R31. doi: 10.1186/cc5713. [PubMed: 17331245].

22. Martí-Carvajal AJ, Dayer M, Conterno LO, Gonzalez Garay AG, Martí-Amarista CE,Simancas-Racines D. A comparison of different antibiotic regimens for thetreatment of infective endocarditis. Cochrane Database Syst Rev. 2016;4:CD009880. doi: 10.1002/14651858.CD009880.pub2. [PubMed: 27092951]. 